\title{
Cmambu
}

\section{СТРАТЕГИИ ПОДДЕРЖАНИЯ СОЦИАЛЬНОЙ ИДЕНТИЧНОСТИ: ЗНАЧЕНИЕ ДЛЯ ТЕОРИИ СОЦИАЛЬНОЙ ИДЕНТИЧНОСТИ И ПОНИМАНИЯ МЕЖГРУППОВЫХ ОТНОШЕНИЙ}

\begin{abstract}
M.B. KOTOBA ${ }^{\mathrm{a}}$
${ }^{a}$ Национальный исследовательский университет «Высшая школа экономики», 101000, Россия, Москва, ул. Мясниикая, д. 20

\section{Резюме}

В статье рассматривается понятие «стратегии поддержания социальной идентичности» и обосновывается его значение для теории социальной идентичности и понимания межгрупповых отношений. Исходной точкой рассуждения являются противоречия, выявляемые при изучении взаимосвязей идентификации с группой, ингруппового фаворитизма, отношения к аутгруппам и самооценки. Для поиска причин возникающих противоречий и возможного способа их решения в статье проведен анализ методологических и эмпирических исследований в рамках теории социальной идентичности и в смежных областях. На этом основании автор заключает, что, во-первых, в теории социальной идентичности неявно существуют две позиции относительно понимания идентичности, что может вести к противоречиям в получаемых данных: с одной стороны, идентичность рассматривается лишь как промежуточное звено между процессами категоризации и сравнения, с другой - как самостоятельный феномен, для поддержания которого в теории выделяется целый набор стратегий. Во-вторых, стратегии поддержания идентичности указывают на функции, которые идентичность выполняет как для индивида, так и для группы, хотя в теории идентичность рассматривается только с точки зрения функций, выполняемых для индивида. Смещение фокуса внимания на функции, выполняемые для группы, позволяет взглянуть на исходные противоречия с новой позиции. В первую очередь, признание того факта, что идентичность выполняет функции для сохранения группы, ставит под сомнение необходимость индивидуальной позитивной самооценки как единственной конечной «цели» реализации стратегий поддержания идентичности, как это обычно предполагается в исследованиях в рамках
\end{abstract}

Статья подготовлена в ходе проведения исследования в рамках Программы фундаментальных исследований Национального исследовательского университета «Высшая школа экономики» (НИУ ВШЭ) и с использованием средств субсидии в рамках государственной поддержки ведущих университетов Российской Федерации «5-100». 
теории социальной идентичности. Также возникает вопрос о том, «за счет чего или кого» поддерживается позитивность идентичности при межгрупповых сравнениях. Соответственно стратегия, выбираемая членами группы для поддержания идентичности, может более или менее позитивно влиять на отношение к аутгруппам, что делает стратегии поддержания идентичности важным объяснительным понятием при изучении межгрупповых отношений.

Ключевые слова: теория социальной идентичности, межгрупповые отношения, стратегии поддержания идентичности, функции социальной идентичности.

\section{Введение. Постановка проблемы исследования}

Методологические работы по истории исследования социальной идентичности указывают, что она далеко не всегда выполняет ту роль в межгрупповых отношениях, которая изначально ей отводилась в теоретических построениях социальных психологов (Brewer, 1991, 2010; Brown, 2000; Ellemers et al., 1997). B первую очередь это касается взаимосвязи идентичности с ингрупповым фаворитизмом, отношением к аут-

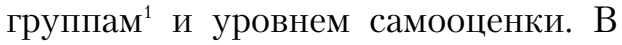
частности, согласно теоретическим предположениям, выраженная идентичность с группой должна вести к увеличению ингруппового фаворитизма, поддерживать позитивную самооценку и приводить к снижению благоприятного отношения к другим группам. Однако обзоры исследований показывают, что это не так, взаимосвязи между указанными феноменами могут меняться на противоположные или исчезать вовсе (см., например: Brewer, 1999, 2010; Brown, 2000).

Разумеется, выявленные противоречия не остались без внимания исследователей, были предложены различные способы их разрешения, например, теория оптимальной отличительности М. Бруэр (Brewer, 1991), модель Хинкля-Брауна и др. (для обзора см.: Brown, 2000). Тем не менее, с нашей точки зрения, предложенные решения не учитывают две особенности теории социальной идентичности. Во-первых, анализ показывает, что в теории социальной идентичности существует несколько положений относительно идентичности, не связанных в единое целое, что ведет к противоречиям в эмпирических исследованиях. Во-вторых, теория социальной идентичности содержит идею о существовании стратегий поддержания идентичности. Потенциал этой идеи для понимания взаимосвязей идентичности и других феноменов межгрупповых отношений практически не рассматривался ранее.

${ }^{1}$ В данной работе словосочетание «ингрупповой фаворитизм» является транскрипцией англоязычного термина «ingroup favoritism», подразумевающего предпочтение своей группы (группы принадлежности) перед другим группам (аутгруппам) при распределении благ. «Аутгруппа» является заимствованием термина «outgroup», обычно относящегося к обозначению иных групп, членом которых человек не является. 
Мы полагаем, что стратегии поддержания идентичности могут указывать на функции, которые идентичность выполняет как для человека, так и для группы в рамках межгрупповых отношений. Несмотря на существование теоретических положений о функциональной взаимосвязи идентичности и различных феноменов - например, потребности в принадлежности к группе (Baumeister, Leary, 1995) или самооценки (Tajfel, 1982), - социальная идентичность систематически не обсуждается с позиций функционального анализа. Еще меньшее внимание получает разделение функций идентичности на выполняемые для индивида и для группы. Однако, как нам представляется, учет каждого из типов функций позволяет предложить новое решение проблемы взаимосвязи идентичности и самооценки, с одной стороны, и идентичности, ингруппового фаворитизма и отношения к аутгруппам - с другой. Подробная логика обоснования высказанных здесь суждений приведена далее. Сначала мы проанализируем двойственное понимание идентичности в теории социальной идентичности, а затем обсудим значение стратегий поддержания идентичности для решения указанных выше проблем.

\section{Понимание идентичности как центральный «камень преткновения» теории социальной идентичности}

Теория социальной идентичности (Social Identity Theory, SIT), paзpaботанная А. Тэшфелом с соавт., создала основу взгляда на идентичность как на результат осознания человеком своего членства в группе и как на минимальное условие для возникновения межгруппового поведения (Brewer, 2010; Brown, 2004). Теория стала, пожалуй, одной из наиболее влиятельных в изучении межгруппового поведения, в первую очередь, благодаря продуктивным идеям о том, как изучать поведение человека, учитывая одновременно индивидуальный и групповой уровни (Brown, 2000, 2004).

Чаще всего в контексте межгрупповых отношений указываются два положения SIT, которые будут важны нам для дальнейшего анализа: стремление к «позитивной отличительности» (positive distinctiveness) и роль социальной категоризации в формировании межгруппового поведения (Brewer, 1991; Brown, 2000; Ellemers et al., 1997; Tajfel, 1982). Первое положение: человеку свойственно стремление к позитивной (за редким исключением) Я-концепции и, как следствие, к позитивной социальной идентичности (Tajfel, 1982). Позитивность Я-концепции человека существенно зависит от позитивности оценки группы принадлежности. Высокая оценка ингруппы влияет на собственную самооценку человека и позволяет погреться в «лучах славы» группы, что не раз подтверждалось эмпирически (Brewer, 2010). За счет чего, согласно SIT, возможна позитивная отличительность? А. Тэшфел предлагает модель, которую он обозначил как «C.I.C. theory» (categorization-identificationcomparison, или категоризацияидентификация-сравнение), где указанные три процесса ведут к возможности позитивной отличительности 
своей группы (Tajfel, 1982). В этой модели сравнение становится своеобразным «конечным продуктом» процессов категоризации и идентификации. Именно через сравнение достигается «позитивная отличительность группы» («positive group distinctiveness»), так необходимая для позитивной оценки группы и, собственно, для позитивной социальной идентичности (Ibid.).

Второе положение SIT, которое важно с точки зрения межгруппового поведения, касается категоризации как механизма социального познания. Категоризация в приведенной выше модели является начальным процессом, который делает возможным возникновение межгруппового поведения и выступает его минимальным условием, что показали А. Тэшфел с соавт. в эксперименте, где участники практически номинальных групп проявляли межгрупповую дифференциацию (Tajfel et al., 1971). Позднее А. Тэшфел и M. Биллиг выяснили, что самого факта указания в инструкции, что участник эксперимента будет входить в «группу», и создания абсолютно случайных групп было достаточно для возникновения ингруппового фаворитизма и стремления к позитивной отличительности (Billig, Tajfel, 1973). Категоризация приводит к преувеличению различий между группами, или категориями, и преуменьшению внутригрупповых различий, или различий элементов одной категории (Tajfel, 1982). Экспериментальное подтверждение было найдено сначала для физических стимулов, а далее и для социальных категорий (Brown, 2004). По сути, следствия категоризации соз- дают группу как психологическую реальность и тесно связаны с восприятием ее как субъекта действия. Это стало предметом целого направления исследований, посвященных воспринимаемой целостности группы и ее следствий для группового поведения и межгрупповых отношений (Abelson et al., 1998; Campbell, 1958; Dasgupta et al., 1999). Таким образом, в рамках SIT был предложен механизм возникновения группового поведения, а также формирования пристрастности по отношению к своей группе в сравнении с аутгруппами. Идеи теории легли в основу большого числа исследований. Но, как и любая плодотворная теория, SIT столкнулась со сложностями, противоречиями в эмпирических данных, полученных при ее применении.

Р. Браун выделяет пять наиболее существенных проблем, которые проявились по мере накопления фактов в рамках SIT, и подробно анализирует историю предложенных решений (Brown, 2000). Во-первых, исследования указывают, что взаимосвязь между идентификацией с группой и ингрупповой пристрастностью может варьироваться от положительной до отрицательной, а также может отсутствовать. Во-вторых, самооценка человека и ее позитивность далеко не всегда играют ту роль, которая им отводится в теории, а именно: роль цели, ради которой человек стремится к позитивной идентичности и, соответственно, позитивной оценке группы принадлежности. В-третьих, был обнаружен достаточно загадочный феномен позитивно-негативной асимметрии отсутствие эффекта ингрупповой 
пристрастности при распределении «наказаний», а не благ. В-четвертых, стремление к позитивной отличительности далеко не всегда приводит к ожидаемому эффекту увеличения неприязни среди похожих групп. В-пятых, пока представлено очень мало данных относительно оснований выбора стратегий поддержания идентичности низкостатусными группами. Разумеется, ни одна из проблем не осталась незамеченной. Были предложены различные решения для каждой из них (Ibid.).

Особняком во всей дискуссии, с нашей точки зрения, стоит ответ Дж. Тернера на критику SIT, который отмечал, что ряд гипотез, в частности необходимости позитивной связи между идентификацией с группой и ингрупповым фаворитизмом или гипотеза самооценки, просто не следуют из теории, поэтому противоречивость их проверки имеет мало отношения к самой теории (Turner, 1999). С одной стороны, Дж. Тернер прав, выдвигая вопрос верифицируемости теории и правомерности ее перевода в те или иные гипотезы для проверки. Как нам представляется, теория предлагает механизм взаимосвязи различных межгрупповых феноменов (идентичности, сравнения, категоризации и их следствий) в «чистом» виде, в некотором роде в вакууме. В пользу такого предположения свидетельствует также и основной методический прием, парадигма минимальных групп (minimal group paradigm), разработанный А. Тэшфелом с соавт. специально для SIT (Tajfel et al., 1971) и также ставший причиной многих споров (Brewer, 1979; Mummendey et al., 2000).
С методологической точки зрения перенесение предложенных закономерностей в другие межгрупповые условия (другие статусные отношения, возможное разнообразие мотивации членства в группе, видов социальной идентичности и т.д.) вовсе не должно приводить к их точному повторению (Левин, 2001). Напротив, взаимосвязи или феноменология должны меняться, подобно тому как перышко и камень различным образом падают в условиях вакуума и воздушной среды, что не отменяет одинакового действия закона тяготения. Можно утверждать, что разнообразные следствия из теории учитывали одни факторы контекста, но не учитывали другие. Соответственно, их подтверждение или отрицание без учета закономерностей функционирования идентичности мало что говорит о положениях теории. Но, с другой стороны, нельзя не принять логику Р. Брауна, который в ответ на критику Дж. Тернера показывает, что именно подобного рода гипотезы должны были последовать из основных положений SIT (Brown, 2000). Само существование этого спора указывает, что SIT содержит суждения, которые могут в ряде случаев входить в противоречие или оставлять возможность для различных дальнейших трактовок.

На наш взгляд, наиболее вероятными кандидатами быть «камнем преткновения», как ни парадоксально, выступают стремление к позитивности самооценки и понимание идентичности, т.е. центральные составляющие теории. SIT не единожды критиковалась за излишнюю «когнитивность» и невнимание к эмоциональным и мотивационным 
процессам (Ibid.). В теории действительно точнее прописаны когнитивные механизмы межгрупповых отношений (в особенности категоризация и ее следствия) и существенно меньше внимания уделяется эмоциональным и мотивационным процессам. Однако, как нам кажется, проблема сложнее, поскольку, несмотря на общую «когнитивность» теории, А. Тэшфел основывается именно на мотивационных причинах поведения: стремлении к позитивной самооценке. С позитивностью самооценки, позитивностью оценки ингруппы и стремлением к позитивной отличительности связано большинство проблем теории, выделенных Р. Брауном (Ibid.).

C нашей точки зрения, стремление к позитивности самооценки, или к позитивной отличительности, вполне может быть подвергнуто сомнению, тем более, что основания и фундаментальность этого стремления человека и его необходимость в теории детально не обсуждаются. Например, М. Бруэр предложила достаточно продуктивную теорию оптимальной отличительности, с точки зрения которой социальное Я человека (social self) с необходимостью связано с двумя противоположными потребностями - стремлением принадлежать к чему-то большему, к группе, и стремлением быть отдельной и уникальной личностью (Brewer, 1991). Для человека одинаково некомфортны ситуации, где баланс между этими стремлениями нарушается в ту или иную сторону: слишком «растворенность» в группе или слишком «отдельность» от группы. По мысли М. Бруэр, только в случае достижения оптимальной отличительности «Я-группа» социальная идентичность и лояльность к группе будут связаны позитивно. При возникновении дисбаланса связь будет иной (Ibid.).

Кросс-культурный анализ ставит под сомнение универсальность стремления к позитивной самооценке как таковой. С. Хайне с соавт. на основании сравнительного анализа культурных особенностей и эмпирических исследований самооценки стран Северной Америки и Японии убедительно показывают, что концепция самооценки (self-esteem) в принципе сужает существующую реальность вариантов самопонимания и самоотношения человека (selfregard). Авторы считают, что, например, для японца не свойственно самоотношение, базирующееся на позитивности самооценки по накопительному признаку (чем выше, тем лучше), в отличие от жителя стран Северной Америки или Европы (Heine et al., 1999). При этом Япония представляет ярко выраженную коллективистическую культуру. Тогда придется признать, что мотивация членства в группе для японца должна быть другой, нежели позитивность самооценки.

Если возвратиться к модели Тэшфела («C.I.C. theory»), то есть основания предположить, что наименее изученный процесс в ней - это «середина», или идентичность (identification). Равно как и «позитивность», она скорее постулируется как необходимый элемент цепочки процессов: если есть группа принадлежности, значит, есть идентичность. Идентификация и идентичность как ее результат зачастую рассматриваются в качестве само собой разумеющегося 
состояния, которое может быть больше или меньше выражено по ряду параметров. Причем понимание идентичности как определенного статуса или состояния свойственно не только SIT. Наиболее известная модель этнической идентичности Дж. Финни (Phinney, 1990), несмотря на то, что она создавалась для оценки развития идентичности, не раз критиковалась именно за отсутствие возможности оценить развитие с помощью предложенной технологии (Umaña-Taylor et al., 2004). В детальном и объемном обзоре Р. Эшмор с соавт. (Ashmore et al., 2004) выделили около десятка основных измерений, по которым изучается социальная, или коллективная, идентичность, однако все они описывают идентичность как некоторый результат идентификации без учета путей ее становления.

Соответственно, теория социальной идентичности содержит положения, которые не могут являться исходными для выведения последующих гипотез (и их эмпирической проверки), поскольку сами требуют отдельного обоснования. Например, не очень логично ожидать систематической позитивной взаимосвязи между уровнем самооценки и межгрупповой дифференциацией (что стало предметом исследований нескольких десятилетий), так как сомнительна сама по себе базовость стремления к позитивной самооценке. Также нелогично располагать идентичность как элемент уравнения между категоризацией (или самокатегоризацией) и межгрупповым сравнением и ожидать линейных взаимосвязей, поскольку сомнителен тот факт, что идентичность - лишь автоматический результат принадлежности к группе.

Тем не менее одновременно со взглядом на идентичность, подвергнутым критике выше, SIT содержит и другое положение относительно идентичности, которое, на наш взгляд, способно как вернуть в теорию «динамичность» идентичности и контекст межгрупповых сравнений, так и поставить под сомнение логику основных процессов теории (категоризация - идентификация сравнение). Мы имеем в виду идею о стратегиях поддержания идентичности, т.е. определенных действий, при помощи которых человек или группа восстанавливает позитивность идентичности при неблагоприятном межгрупповом сравнении (Tajfel, 1982). Стратегии поддержания идентичности, с нашей точки зрения, указывают на возможное решение найденных противоречий во взаимосвязях идентичности и других феноменов межгрупповых отношений. Далее мы рассмотрим подробнее понятие стратегий поддержания идентичности и его возможное значение как для SIT, так и для понимания межгрупповых отношений.

\section{Стратегии поддержания идентичности: понятие, роль для SIT и последствия для понимания межгрупповых отношений}

Стратегии, при помощи которых человек может поддерживать позитивность идентичности, подразделялись в рамках SIT на три группы: индивидуальная мобильность, социальное творчество и социальное соревнование (Tajfel, 1982). Важно отметить, что А. Тэшфел подчеркивает 
приоритетную роль специфики сложившихся отношений между двумя группами для выбора стратегии: «Природа существующих отношений между группами определяет выбор одной или нескольких стратегий из доступных этнолингвистическому меньшинству для того, чтобы справиться с невыгодным положением» (Ibid., p. 26), хотя контекст межгрупповых отношений редко становился предметом отдельного внимания исследователей (Verkuyten, Reijerse, 2008).

Индивидуальные стратегии мобильности, такие как ассимиляция (assimilation) и «незаконная» ассимиляция («illegitimate» assimilation), используются человеком в том случае, когда групповые границы проницаемы и человек просто может «выдать себя» за представителя большинства, например, изучив язык, сменив имя, свою персональную историю и т.п. Коллективные стратегии появляются, когда нет возможности легко проникнуть в высокостатусную группу и/или когда идентичность группы принадлежности очень сильна и человек не может от нее «отказаться». В таком случае развиваются стратегии, названные А. Тэшфелом и Дж. Тернером «социальным творчеством» (social creativity) и социальным соревнованием (social competition). Для описания выделенных за последние три десятилетия стратегий можно обратиться к таксономии, предложенной М. Бланцем с соавт., поскольку она наиболее исчерпывающая (Blanz et al., 1998). После ряда эмпирических исследований они выделили двенадцать стратегий, которые составляют шесть групп, на основании двух измерений (изменение параметров межгруппового сравнения и тип реакции). Наглядное распределение стратегий по группам и измерениям можно увидеть на рисунке 1.

Рисунок 1

Таксономия стратегий поддержания позитивности идентичности (приводится по: Blanz et al., 1998, p. 722)

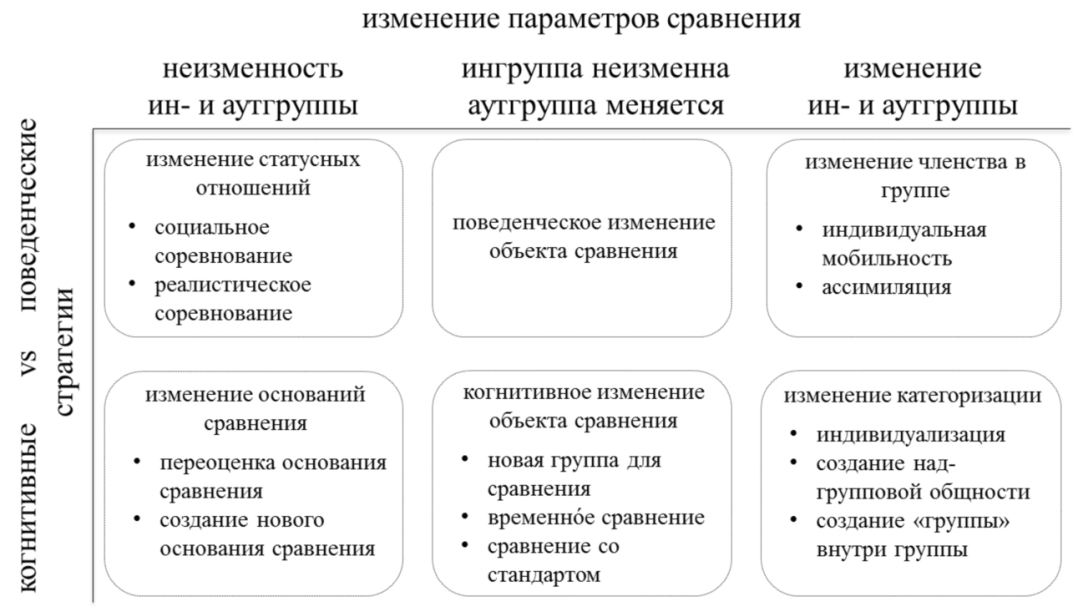


Первую группу стратегий отсутствие изменений ин- и аутгруппы на поведенческом уровне - составляют стратегии социального и реалистического соревнования (Social competition and Realistic competition). Социальное соревнование заключается в своеобразном состязании по убеждению себя и окружающих, что «мы лучшие» в разных сферах: занимаемых позициях, наличии талантов и т.п. Реалистическое соревнование - активное участие в социальных движениях для изменения статуса группы. Еще в 1979 г. Дж. Тернер, Р. Браун и А. Тэшфел предположили, что мотивация, стоящая за реалистическим и социальным соревнованием, различна (Turner et al., 1979). Реалистическое соревнование, в первую очередь, вызывается материальными причинами, а социальное соревнование - релевантностью аутгруппы, т.е. восприятием групп (своей и другой) как сравнимых между собой. Когнитивная реакция при отсутствии изменений ин- и аутгруппы (вторая группа стратегий) реализуется в изменении сути сравнения, т.е. в стратегиях переоценки оснований сравнения (Reevaluation of comparison dimension) и создании нового основания (New comparison dimension). Например, члены группы оценивают себя как «добрых и гостеприимных», а другую группу как «деловитых и предприимчивых», только доброта гораздо выше стоит в ценностях группы, нежели деловитость - такой эффект был получен при исследовании молодежи в 1980-е гг. в СССР и ее отношения к Coeдиненным Штатам (Стефаненко, 2014).

Следующие две группы стратегий связаны с изменением аутгруппы при неизменной ингруппе. На уровне поведения человек может изменить объект сравнения. Предположительно такая стратегия должна реализоваться в попытках разными способами изменить статус аутгруппы, при неизменном статусе собственной группы. Однако эта стратегия эмпирически не измерялась авторами и является гипотетическим дополнением получившейся модели. Когнитивные стратегии в этом кластере - выбор новой группы для сравнения (New comparison group), временно́е сравнение (Temporal comparison) и сравнение со стандартом (Comparison with standard). Выбор новой группы позволяет сменить фокус сравнения и выбрать ту группу, которая находится еще в более уязвимом состоянии, чем своя собственная. Соответственно, своя группа на этом фоне выглядит более привлекательно. Временно́е сравнение это сравнение не с нынешним положением группы, а с прошлым временем благополучного состояния. Например, человек, переехавший в другую страну, оценивает свою этническую группу не как уязвимую группу мигрантов, а опирается на ее статус в родной стране. Сравнение со стандартом позволяет преодолеть относительность сравнения и установить стандарты, перед которыми «все равны». Например, гарантированный законом доступ к благам, равные возможности или универсальные ценности как основа сравнения.

И последние две группы стратегий - описывают изменение как ин-, так и аутгруппы. На поведенческом уровне это уже упоминавшиеся выше стратегии индивидуальной 
мобильности и ассимиляции. На когнитивном - индивидуализация (Individualization), создание надгрупповой общности (Superordinate re-categorization) и создание «группы» внутри группы (Subordinate re-categorization). Индивидуализация заключается в том, что человек как бы выделяет себя из группы, не считая, что ее статус как-либо должен отражаться на нем. Яркий пример подобного разотождествления себя и своей этнической группы можно найти в исследовании В.С. Собкина. Среди еврейских подростков в 1990-е гг. была обнаружена тенденция весьма пессимистично оценивать перспективы евреев как этноса в России, но при этом же возрастал оптимизм в оценке своих собственных перспектив (Собкин, 1995).

C нашей точки зрения, само по себе существование стратегий поддержания идентичности (и, разумеется, эмпирические данные, указывающие на действительное их разнообразие) может способствовать пониманию ряда проблем SIT. Вопервых, логично предположить, например, что при стратегиях индивидуализации и индивидуальной мобильности соотношение между выраженностью идентичности с группой и ингрупповой пристрастностью будет иным, нежели при поиске новых оснований для межгруппового сравнения, или при временно́м сравнении и сравнении со стандартом. Поскольку стратегии индивидуализации и индивидуальной мобильности указывают на отрицание значимости принадлежности к данной группе, то идентичность с этой группой не должна быть тесно связана с ингрупповой при- страстностью, самооценкой или отношением к аутгруппам. Напротив, при «сравнительных» стратегиях идентичность должна быть связана с ингрупповым фаворитизмом, хотя связь с отношением к аутгруппам может быть различной, в зависимости о того, какая сравнительная стратегия избрана человеком или группой. Также стратегии должны задавать различные основания для позитивности самооценки, что может «разрывать» обязательность связи между необходимостью позитивной оценки своей группы и позитивностью самооценки. Например, предпочтение индивидуальной мобильности указывает на то, что человек уже ориентирован на другую группу, нежели группа членства, соответственно, в таком случае ингруппа (позитивность оценки которой при этом измеряется эмпирически) может и не являться источником позитивной самооценки.

Во-вторых, признание существования различных стратегий, при помощи которых человек может поддерживать позитивность своей идентичности, приводит к вмешательству в основную цепочку процессов SIT. Например, такая стратегия, как индивидуализация, подразумевает отрицание важности межгруппового сравнения как такового и социальной идентичности в целом для поддержания самооценки, хотя бы на одном из этапов принадлежности человека к группе. Это же свойственно и сравнению со стандартом, только в данном случае отрицается не сравнение само по себе, а межгрупповое сравнение. Создание «надгрупповой» общности и «группы» внутри группы не меняет логики 
модели C.I.C., но подразумевает обязательным следующий компонент рекатегоризацию (recategorization), прежде чем будет достигнута позитивная отличительность.

В-третьих, существование стратегий поддержания идентичности почти с необходимостью ведет к вопросу: что является основанием выбора той или иной стратегии? Этот вопрос составляет пятую из проблем SIT, выделяемых Р. Брауном, и вносит динамический аспект в понимание идентичности в противовес исключительно когнитивному (Brown, 2000). Пожалуй, наиболее систематичный ответ на этот вопрос, причем с точки зрения специфики межгрупповых отношений, на чем акцентировал внимание еще А. Тэшфел, дают исследования М. Веркайтена с соавт. Они выделяют три параметра межгруппового сравнения, важных для оценки межгрупповых отношений: воспринимаемые стабильность статуса группы, проницаемость межгрупповых границ, легитимность иерархии статусов (Verkuyten, Reijerse, 2008). Различное сочетание этих факторов влечет за собой предпочтение той или иной стратегии поддержания идентичности (например, покинуть группу или остаться в ней), а также указывает на причины различий в восприятии межгрупповых отношений со стороны группы большинства и меньшинства. Исследования показали, что, например, для группы меньшинства восприятие межгрупповых отношений как легитимных и стабильных сказывается на роли воспринимаемой проницаемости границ: в таком случае она будет негативно связана с ингрупповой идентичностью, но позитивно - с надгрупповой идентичностью и будет вести к ингрупповому фаворитизму по отношению к другим этническим меньшинствам в стране. А для группы большинства то же сочетание будет вести к увеличению ингрупповой идентичности (Ibid.).

Недавнее метааналитическое исследование, посвященное роли культуры в проявлении ингруппового фаворитизма (Fischer, Derham, 2016), также косвенно указывает на важность понимания различных стратегий поддержания идентичности. Анализ данных почти 20 тысяч респондентов из 18 культурных групп показал, что на выраженность ингруппового фаворитизма значимое влияние оказывает уровень избегания неопределенности. Авторы предполагают, что за счет усиления меры предпочтения своей группы человек избегает неопределенности, свойственной сложному социальному миру (Ibid.). Учитывая относительную стабильность уровня избегания неопределенности, присущую культуре, можно предположить, что эта характеристика работает как своеобразная стратегия поддержания позитивной идентичности с группой и ведет к усилению ингруппового фаворитизма. Итак, можно говорить, что стратегии поддержания идентичности указывают на необходимость учета процесса, который приводит к позитивной идентичности. В этом процессе участвует гораздо большее число факторов, нежели только факт исходной категоризации на группы и стремление к позитивной самооценке.

Последнее и самое существенное, на чем стоит остановиться при описании потенциала понятия стратегий 
поддержания идентичности, - на предположении, что стратегии поддержания идентичности могут указывать на функции идентичности, которые она выполняет как для индивида, так и для группы. Что касается функций идентичности, которые она выполняет для индивида, то это предположение практически напрямую следует из SIT, так как по теории позитивность идентичности - лишь промежуточное звено в цепи процессов, приводящих к поддержанию Я-концепции человека в целом (Tajfel, 1982). Можно утверждать, что большинство исследований стратегий поддержания идентичности, несмотря на анализ групповых процессов, явно или неявно отталкиваются от функций идентичности для отдельного человека. Это наиболее явственно для так называемых индивидуальных стратегий (индивидуальная мобильность, ассимиляция), поскольку они скорее отрицают идентификацию с изначальной группой принадлежности, нежели направлены на ее поддержание, что подтверждается и эмпирически. Также одна из немногих систематичных находок относительно выбора стратегии заключается в том, что чем меньше выражена идентичность с группой, тем вероятнее выбор стратегии индивидуальной мобильности (Brown, 2000). Но и другая группа стратегий, «коллективные» (или социального творчества), с нашей точки зрения, также изучалась с позиции существующих функций идентичности для индивида, поскольку, согласно теории, человек ищет способы поднять статус группы именно для того, чтобы поддержать свою Я-концепцию. Соответ- ственно, повышение группового статуса при помощи стратегий социального творчества - лишь необходимость, так как человек не может выйти из своей группы и присоединиться к группе с высоким статусом, тогда как конечная цель всего процесса индивидуальная самооценка.

Однако два наблюдения не позволяют нам остановиться на выводе, что все стратегии, предложенные в теории, свидетельствуют о функциях идентичности для индивида. Во-первых, анализ проведенных исследований показывает, что те или иные стратегии социального творчества выбираются представителями групп не хаотично (по личным предпочтениям), но существуют различия между группами большинства и меньшинства (Niens, Cairns, 2002; Ellemers, Rijswijk, 1997; Verkuyten, Reijerse, 2008), разного статуса (Ellemers, Rijswijk, 1997; Mummendey et al., 1999), с разной историей межгрупповых отношений (Dumont, Waldzus, 2015). Это указывает на существование надындивидуальных факторов, определяющих выбор стратегии. Во-вторых, определенная традиция исследований указывает на тот факт, что социальные группы и границы групп конструируются и поддерживаются социальными, а не биологическими силами (Барт, 2006; Зивертс, 2006; Котова, 2010; Стефаненко, 1999). Но при отсутствии объективных жестких групповых границ возникает вопрос: за счет чего сохраняются границы группы и за счет чего сохраняется групповая целостность?

Этот вопрос становится еще более насущным, если учесть, что пока не существует социальных структур, 
где все группы обладали бы равным статусом, однако нельзя утверждать, что группы с относительно более низким статусом обречены на исчезновение и растворение в группе большинства. Скорее происходят обратные процессы, и группа большинства, и группа меньшинства сопротивляются взаимному перемешиванию или даже установлению надгрупповых общностей. Об этом свидетельствуют, например, исследования успешности принятия политики мультикультурализма (Berry et al., 2006; Sam, Berry, 2010; Verkuyten, Yildiz, 2007), проблем формирования надгрупповой идентичности (Brewer, 2010). И если группа не распадается при неблагоприятном межгрупповом сравнении, особенно при стабильности невысокого статуса во времени, то должны существовать силы, позволяющие поддерживать привлекательность группы в такой ситуации и восприятие границ группы как относительно непроницаемых. С нашей точки зрения, один из вероятных кандидатов на роль такой силы - социальная идентичность, которая способна выполнять функцию сохранения группы, даже при неблагоприятном межгрупповом сравнении. Разумеется, идея о том, что социальная идентичность способствует сохранению группы, не нова. Близкую идею высказывал Ф. Барт еще полвека назад (Барт, 2006), достаточно много доказательств собрано о важности этнической, гражданской, религиозной идентичности и о роли этих групп принадлежности для человека и для общества (Стефаненко, 2014). Однако применение идей функционального анализа к пониманию идентичности систематически не проводилось. Между тем с точки зрения функционального анализа признание того факта, что идентичность выполняет социальные функции, влечет за собой ряд следствий. Эти следствия напрямую касаются исходной проблемы нашего обсуждения - взаимосвязи идентичности, самооценки, ингруппового фаворитизма и отношения к аутгруппам.

Согласно положениям функционального анализа, элемент (в данном случае идентичность) выполняет свою функцию для определенной системы (группы), причем ключевым является значение элемента именно для системы, ее благополучия (Козер, 2000; Ariew et al., 2002). В этом смысле своеобразная «цель» идентичности заключается именно в сохранении группы, при том, что самооценка (как показатель субъективного благополучия) каждого члена группы не обязательно будет расти в этом процессе. С этой точки зрения, обычно полагаемая необходимой связь между идентичностью и индивидуальной самооценкой перестает быть таковой. Напротив, идентичность и приверженность человека группе должны опираться на иные основания, нежели поддержание самооценки, к такому выводу приходят, например, С. Хайне с соавт. (Heine et al., 1999). Более понятными становятся и результаты исследований процессов аккультурации: с одной стороны, существует представление, что стремление группы к сепарации обычно связано с уменьшением субъективного благополучия, с другой стороны - нередки примеры, когда группы систематически придерживаются стратегии сепарации 
(Sam, Berry, 2010; Verkuyten, Yildiz, 2007). С позиции функционального анализа данные примеры - результат проявления социальной функции идентичности (сохранение границ группы), несмотря на возможные неблагоприятные последствия для индивидуального благополучия.

Подробное рассмотрение функций социальной идентичности предмет отдельного анализа, выходящий за рамки данной статьи. Здесь мы приведем лишь несколько примеров, чтобы показать, каким образом стратегии поддержания идентичности могут способствовать изучению ее функций. C нашей точки зрения, стратегии поддержания идентичности указывают, как именно и за счет чего идентичность выполняет свои функции, а также позволяют посмотреть на различные феномены межгрупповых отношений с новой точки зрения. Например, Дж. Бекер провела недавно исследование и в серии экспериментов показала, как возможность воспользоваться одной из стратегий социального творчества (изменить основание сравнения или группу сравнения) приводит к уменьшению желания участвовать в общественных акциях за равенство прав (Becker, 2012). Автор обсуждает полученные результаты скорее с негативной точки зрения и приходит к мысли, что стратегии социального творчества способствуют консервации неравенства и замедлению борьбы за равенство прав. Однако, по нашему мнению, за рамками рассмотрения осталась позитивная роль стратегий социального творчества, которая в данном исследовании про- явилась довольно явно: позволяя поддержать позитивную идентичность альтернативным способом, нежели прямая борьба, они способствуют сохранению сложившейся социальной структуры, разрушение которой может вести к непредсказуемым последствиям как для группы, так и для отдельного человека.

Другой пример связан с переоценкой роли социальной идентичности в межгрупповых отношениях. Внимание к функциям идентичности для группы позволяет сместить фокус анализа от привычной постановки проблемы через соотношение меры приверженности к своей группе и позитивности или негативности отношения к аутгруппам к новому вопросу: за счет чего поддерживается эта мера приверженности. Одна ситуация, когда привлекательность группы поддерживается за счет «внутренних ресурсов»: культурных, политических и иных достижений, другая - когда для привлекательности группы необходимо принизить статус другого, в данном случае другой группы. Своеобразную параллель можно провести с исследованиями гражданской идентичности, где есть эмпирические свидетельства существования двух ее видов: патриотизма («внутренние ресурсы») и национализма (сравнение с другими). Причем взаимосвязь патриотизма и национализма может быть различной и каждый из этих видов идентичности отличным образом связан с позитивным отношением к аутгруппам (мигрантам): патриотизм обычно связан положительно, а национализм - отрицательно (Grigoryan, 2016). 


\section{Заключение}

В данной работе мы рассмотрели роль стратегий поддержания идентичности для теории социальной идентичности и для межгрупповых отношений. Обобщая проведенный анализ, можно кратко представить его результат в виде нескольких положений. Во-первых, стратегии поддержания идентичности позволяют точнее оценивать взаимосвязь между идентичностью, индивидуальной самооценкой, ингрупповым фаворитизмом и отношением к аутгруппам. Они «отрицают» однозначную последовательность процессов модели C.I.С. и предполагают разнообразие факторов, которые могут выступить медиаторами или модераторами взаимосвязей. Во-вторых, стратегии поддержания идентичности указывают на функции, которые идентичность может выполнять для индивида и для группы, и служат способами выполнения этих функций. При этом идея о функциях идентичности для индивида практически напрямую следует из теории социальной идентичности, но положение о функциях идентичности для группы менее очевидным образом связано с теорией и практически не привлекало внимания исследователей. Рассмотрение идентичности с точки зрения ее функций для группы приводит к ряду следствий. Первое следствие связано с исчезновением необходимости считать стремление к позитивной самооценке основной причиной поддержания идентичности, второе следствие касается воз- можности переосмыслить ряд феноменов межгрупповых отношений и данные исследований. Таким образом, понятие стратегий поддержания идентичности, с нашей точки зрения, является крайне продуктивным как для понимания принципов функционирования идентичности, так и для решения противоречий, возникающих в теории социальной идентичности.

Как нам представляется, дальнейшим этапом теоретического рассмотрения должен стать, с одной стороны, анализ редких примеров применения функционального анализа в межгрупповых отношениях: изучение функций групп (Brown, 2000), социальных функций предубеждений (Котова, 2010). С другой стороны - обобщение примеров рассогласования теоретически ожидаемого группового поведения, направленного на достижение большего субъективного благополучия, и данных эмпирических исследований. Такие факты накапливаются в рамках изучения процессов аккультурации (см., например: Berry et al., 2006; Sam, Berry, 2010) и могут свидетельствовать о проявлении функций идентичности для группы. С эмпирической точки зрения приобретают значимость анализ существующих технологий измерения стратегий поддержания идентичности (Blanz et al., 1998; Ellemers, Rijswijk, 1997; Verkuyten, Reijerse, 2008), их адаптация в российском контексте и разработка экспериментальных исследований для изучения функций идентичности, которые она выполняет для индивида и для группы. 


\section{Литература}

Барт, Ф. (2006). Введение. В кн. Ф. Барт (ред.), Этнические группы и социальные границы. Социальная организация культурных различий. Сборник статей (с. 9-48). М.: Новое изд-во.

Зивертс, Х. (2006). Этническая стабильность и динамика границ в южной Мексике. В кн. Ф. Барт (ред.), Этнические группы и сочиальные гранищы. Социальная организация культурных различий. Сборник статей (с. 122-141). М.: Новое изд-во.

Козер, Л. (2000). Функции социального конфликта. М.: Идея-Пресс/Дом интеллектуальной книги.

Котова, М. В. (2010). Роль предубеждения в восприятии границ этнической группы. Альманах современной науки и образования, 43(12), 130-134.

Левин, К. (2001). Переход от аристотелевского к галилеевскому способу мышления в биологии и психологии. В кн. Д. А. Леонтьев, Е. Ю. Пятаева (ред.), Динамическая психология: Избранные труды (с. 54-84). М.: Смысл.

Собкин, В. С. (1995). Сравнительный анализ особенностей ценностных ориентаций русских и еврейских подростков. В кн. Ценностно-нормативнье ориентации старшеклассника (с. 6-63). М.: ЦСО РАО.

Стефаненко, Т. Г. (1999). Социальная психология этнической идентичности (Неопубликованная докторская диссертация). МГУ им. М.В. Ломоносова, Москва.

Стефаненко, Т. Г. (2014). Этнопсихология: Уиебник (5-е изд., испр. и доп.). М.: Аспект Пресс.

Abelson, R. P., Dasgupta, N., Park, J., \& Banaji, M. B. (1998). Perceptions of the collective other. Personality and Social Psychology Review, 2(4), 243-250.

Ariew, A., Cummins, R., \& Perlman, M. (Eds.). (2002). Functions: new essays in the philosophy of psychology and biology. Oxford: Oxford University Press.

Ashmore, R. D., Deaux, K., \& McLaughlin-Volpe, T. (2004). An organizing framework for collective identity: Articulation and significance of multidimensionality. Psychological Bulletin, 130(1), 80114. doi:10.1037/0033-2909.130.1.80

Baumeister, R. F., \& Leary M. R. (1995). The need to belong: Desire for interpersonal attachments as a fundamental human motivation. Psychological Bulletin, 117(3), 497-529.

Becker, J. C. (2012). The system-stabilizing role of identity management strategies: social creativity can undermine collective action for social change. Journal of Personality and Social Psychology, 103(4), 647-552. doi:10.1037/a0029240

Berry, J. W., Phinney, J. S., Sam, D. L., \& Vedder, P. (2006). Immigrant youth: Acculturation, identity, and adaptation. Applied Psychology: An International Reviere, 55(3), 303-332. doi:10.1111/j.14640597.2006.00256.x

Billig, M., \& Tajfel, H. (1973). Social categorization and similarity in intergroup behavior. European Journal of Social Psychology, 3, 27-52.

Blanz, M., Mummendey, A., Mielke, R., \& Klink, A. (1998). Responding to negative social identity: a taxonomy of identity management strategies. European Journal of Social Psychology, 28(5), 697-729.

Brewer, M. B. (1979). In-group bias in the minimal intergroup situation: A cognitive-motivational analysis. Psychological Bulletin, 86(2), 307-324.

Brewer, M. B. (1991). The Social Self: on being the same and different at the same time. Personality and Social Psychology Bulletin, 17(5), 475-482.

Brewer, M. B. (1999). The psychology of prejudice: ingroup love or outgroup hate? Journal of Social Issues, 55(3), 429-444. 
Brewer, M. B. (2010). Intergroup relations. In R. F. Baumeister \& E. J. Finkel (Eds.), Advanced social psychology: the state of the science (pp. 535-571). Oxford: Oxford University Press.

Brown, R. (2000). Social identity theory: past achievements, current problems and future challenges. European Journal of Social Psychology, 30(6), 745-778.

Brown, R. (2004). Prejudice. Its social psychology. Oxford: Blackwell Publishing.

Campbell, D. T. (1958). Common fate, similarity, and other indices of the status of aggregates as social entities. Behavioral Science, 3, 14-25.

Dasgupta, N., Banaji, M. R., \& Abelson, R. P. (1999). Group entitativity and group perception: associations between physical features and psychological judgment. Journal of Personality and Social Psychology, 77(5), 991-1003. doi:10.1037/0022-3514.77.5.991

Dumont, K. B., \& Waldzus, S. (2015). Ideal selves as identity management strategies. International Journal of Intercultural Relations, 44,1-12. doi:10.1016/j.jintrel.2014.11.003

Ellemers, N., \& van Rijswijk, W. (1997). Identity needs versus social opportunities: the use of group-level and individual-level identity management strategies. Social Psychology Quarterly, 60(1), 52-65.

Ellemers, N., Spears, R., \& Doosje, B. (1997). Sticking together or falling apart: ingroup identification as a psychological determinant of group commitment. Journal of Personality and Social Psychology, 72, 617-626.

Fischer, R., \& Derham, C. (2016). Is in-group bias culture-dependent? A meta-analysis across 18 societies. Springer Plus, 5(70). doi:10.1186/s40064-015-1663-6

Grigoryan, L. (2016, in press). National identity and anti-immigrant attitudes: The case of Russia. In P. Schmidt, J. Grimm, L. Huddy, \& J. Seethaler (Eds.), Dynamics of national identity: Media and societal factors of what we are. London: Routledge.

Heine, S. J., Lehman, D. R., Markus, H. R., \& Kitayama, S. (1999). Is there a universal need for positive self-regard? Psychological Review, 106(4), 766-794.

Mummendey, A., Klink, A., Mielke, R., Wenzel, M., \& Blanz, M. (1999). Socio-structural characteristics of intergroup relations and identity management strategies: Results from a field study in East Germany. European Journal of Social Psychology, 29(2-3), 259-285.

Mummendey, A., Otten, S., Berger, U., \& Kessler, T. (2000). Positive-negative asymmetry in social discrimination: Valence of evaluation and salience of categorization. Personality and Social Psychology Bulletin, 26(10), 1258-1270.

Niens, N., \& Cairns, E. (2002). Identity Management Strategies in Northern Ireland. The Journal of Social Psychology, 142(3), 371-380. doi:10.1080/00224540209603905

Phinney, J. S. (1990). Ethnic identity in adolescents and adults: Review of research. Psychological Bulletin, 108(3), 499-514.

Sam, D. L., \& Berry, J. W. (2010). Acculturation: When individuals and groups of different cultural backgrounds meet. Perspectives on Psychological Science, 5(4), 472-481. doi:10.1177/1745691610373075

Tajfel, H. (1982). Social psychology of intergroup relations. Annual Review of Psychology, 33, 1-39.

Tajfel, H., Flament, C., Billig, M. G., \& Bundy, R. P. (1971). Social categorization and intergroup behavior. European Journal of Social Psychology, 1, 149-178.

Turner, J. C. (1999). Some current issues in research on social identity and self categorization theories. In N. Ellemers, R. Spears, \& B. Doosje (Eds.), Social identity (pp. 6-34). Oxford: Blackwell Publishing.

Turner, J. C., Brown, R. J., \& Tajfel, H. (1979). Social comparison and group interest in ingroup favoritism. European Journal of Social Psychology, 9, 187-204. 
Umaña-Taylor, A. J., Yazedjian, A., \& Bámaca-Gómez, M. (2004). Developing the Ethnic Identity Scale using Eriksonian and social identity perspectives. Identity: An International Journal of Theory and Research, 4(1), 9-38. doi:10.1207/S1532706XID0401_2

Verkuyten, M., \& Reijerse, A. (2008). Intergroup structure and identity management among ethnic minority and majority groups: The interactive effects of perceived stability, legitimacy, and permeability. European Journal of Social Psychology, 38, 106-127. doi:10.1002/ejsp.395

Verkuyten, M., \& Yildiz, A. A. (2007). National (Dis)identification and ethnic and religious identity: A study among Turkish-Dutch muslims. Personal and Social Psychology Bulletin, 33(10), 1448-1462. doi:10.1177/0146167207304276

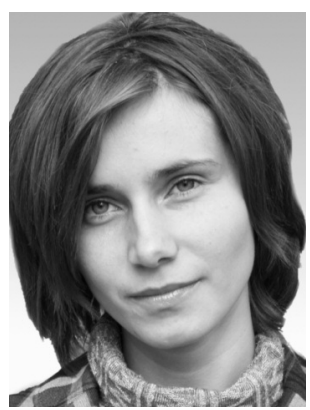

Котова Марина Викторовна - старший преподаватель, факультет социальных наук, департамент психологии, кафедра организационной психологии, Национальный исследовательский университет «Высшая школа экономики», кандидат психологических наук.

Сфера научных интересов: национальная политика и национальные отношения, методы психологического исследования, социальная психология, психология классов и крупных социальных общностей, этнопсихология.

Контакты: mkotova@hse.ru

\title{
Identity Management Strategies: Significance for the Social Identity Theory and Understanding of Intergroup Relations
}

\author{
M.V. Kotova ${ }^{a}$ \\ ${ }^{a}$ National Research University Higher School of Economics, 20 Myasnitskaya str., Moscow, 101000, \\ Russian Federation
}

\begin{abstract}
The present article addresses the notion of identity management strategies. The significance of this concept for development of Social Identity Theory and understanding of intergroup relations is discussed. Contradictions that were found while testing the interrelations between ingroup identification, ingroup favoritism, attitudes toward outgroups, and self-esteem serve as a starting point for the author's reasoning. In order to find causes of such contradictions and possible ways of their solving the author performed theoretical analysis of methodological and empirical research based on Social Identity Theory, as well as functional analysis in related areas. Based on this analysis two suggestions were made. Firstly, Social Identity Theory implicitly contains two meanings of social identity that could lead to discrepancies in empirical results. On the one hand, identity is regarded as just an intermediate element between categorization and comparison, on the other hand, identity is a substantive phenomenon supported by the range of management strategies. Secondly, identity management strategies could specify functions that iden-
\end{abstract}


tity carries out for both an individual and a group. Nevertheless, Social Identity Theory focuses only on those functions that identity carries out for the individual himself. Shifting the focus toward functions that identity executes for a group leads to new suggestions about the initial contradictions. First of all, this leads to doubts in personal self-esteem enhancement as the only "ultimate goal" of strong group commitment. Moreover, this leaves the question open, "at whose expense" the positivity of identity is maintained in the process of intergroup comparisons. Hence, one can expect that while some identity management strategies bring positive outcomes for outgroup attitudes, other strategies bring negative outcomes, which makes identity management strategies a crucial explanatory construct for the study of intergroup relations.

Keywords: social identity theory, intergroup relations, identity management strategies, social identity functions.

\section{References}

Abelson, R. P., Dasgupta, N., Park, J., \& Banaji, M. B. (1998). Perceptions of the collective other. Personality and Social Psychology Review, 2(4), 243-250.

Ariew, A., Cummins, R., \& Perlman, M. (Eds.). (2002). Functions: new essays in the philosophy of psychology and biology. Oxford: Oxford University Press.

Ashmore, R. D., Deaux, K., \& McLaughlin-Volpe, T. (2004). An organizing framework for collective identity: Articulation and significance of multidimensionality. Psychological Bulletin, 130(1), 80114. doi:10.1037/0033-2909.130.1.80

Barth, F. (2006). Vvedenie [Introduction]. In F. Barth (Ed.), Etnicheskie gruppy i sotsial'nye granitsy. Sotsial'naya organizatsiya kul'turnykh razlichii. Sbornik statei [Ethnic groups and social boundaries. The social organization of cultural differences. Collected works] (pp. 9-48). Moscow: Novoe izdatel'stvo. (Transl. of: Barth, F. (1969). Introduction. In F. Barth (Ed.), Ethnic groups and boundaries. The social organization of culture difference (pp. 9-37). Boston: Little, Brown and Company).

Baumeister, R. F., \& Leary M. R. (1995). The need to belong: Desire for interpersonal attachments as a fundamental human motivation. Psychological Bulletin, 117(3), 497-529.

Becker, J. C. (2012). The system-stabilizing role of identity management strategies: social creativity can undermine collective action for social change. Journal of Personality and Social Psychology, 103(4), 647-552. doi:10.1037/a0029240

Berry, J. W., Phinney, J. S., Sam, D. L., \& Vedder, P. (2006). Immigrant youth: Acculturation, identity, and adaptation. Applied Psychology: An International Reviere, 55(3), 303-332. doi:10.1111/j.1464-0597.2006.00256.x

Billig, M., \& Tajfel, H. (1973). Social categorization and similarity in intergroup behavior. European Journal of Social Psychology, 3, 27-52.

Blanz, M., Mummendey, A., Mielke, R., \& Klink, A. (1998). Responding to negative social identity: a taxonomy of identity management strategies. European Journal of Social Psychology, 28(5), 697-729.

Brewer, M. B. (1979). In-group bias in the minimal intergroup situation: A cognitive-motivational analysis. Psychological Bulletin, 86(2), 307-324.

Brewer, M. B. (1991). The Social Self: on being the same and different at the same time. Personality and Social Psychology Bulletin, 17(5), 475-482.

Brewer, M. B. (1999). The psychology of prejudice: ingroup love or outgroup hate? Journal of Social Issues, 55(3), 429-444. 
Brewer, M. B. (2010). Intergroup relations. In R. F. Baumeister \& E. J. Finkel (Eds.), Advanced social psychology: the state of the science (pp. 535-571). Oxford: Oxford University Press.

Brown, R. (2000). Social identity theory: past achievements, current problems and future challenges. European Journal of Social Psychology, 30(6), 745-778.

Brown, R. (2004). Prejudice. Its social psychology. Oxford: Blackwell Publishing.

Campbell, D. T. (1958). Common fate, similarity, and other indices of the status of aggregates as social entities. Behavioral Science, 3, 14-25.

Coser, L. (2000). Funktsii sotsial'nogo konflikta [The functions of social conflict]. Moscow: IdeyaPress/Dom Intellektual'noi Knigi. (Transl. of: Coser, L. (1956). The functions of social conflict. New York: The Free Press).

Dasgupta, N., Banaji, M. R., \& Abelson, R. P. (1999). Group entitativity and group perception: associations between physical features and psychological judgment. Journal of Personality and Social Psychology, 77(5), 991-1003. doi:10.1037/0022-3514.77.5.991

Dumont, K. B., \& Waldzus, S. (2015). Ideal selves as identity management strategies. International Journal of Intercultural Relations, 44, 1-12. doi:10.1016/j.ijintrel.2014.11.003

Ellemers, N., Spears, R., \& Doosje, B. (1997). Sticking together or falling apart: ingroup identification as a psychological determinant of group commitment.Journal of Personality and Social Psychology, 72, 617-626.

Ellemers, N., \& van Rijswijk, W. (1997). Identity needs versus social opportunities: the use of group-level and individual-level identity management strategies. Social Psychology Quarterly, 60(1), 52-65.

Fischer, R., \& Derham, C. (2016). Is in-group bias culture-dependent? A meta-analysis across 18 societies. Springer Plus, 5(70). doi:10.1186/s40064-015-1663-6

Grigoryan, L. (2016, in press). National identity and anti-immigrant attitudes: The case of Russia. In P. Schmidt, J. Grimm, L. Huddy, \& J. Seethaler (Eds.), Dynamics of national identity: Media and societal factors of what we are. London: Routledge.

Heine, S. J., Lehman, D. R., Markus, H. R., \& Kitayama, S. (1999). Is there a universal need for positive self-regard? Psychological Review, 106(4), 766-794.

Kotova, M. V. (2010). Rol' predubezhdeniya v vospriyatii granits etnicheskoi gruppy [The role of prejudice in perception of ethnic group boundaries]. Al'manakh Sozremennoi Nauki i Obrazovaniya, 43(12), 130-134.

Lewin, K. (2001). Perekhod ot aristotelevskogo k galileevskomu sposobu myshleniya v biologii i psikhologii [The transition from Aristotelian to Galilean way of thinking in biology and psychology]. In D. A. Leontiev \& E. Yu. Pyataeva (Eds.), Dinamicheskaya psikhologiya: Izbrannye trudy [The dynamic psychology: Selected works] (pp. 54-84). Moscow: Smysl.

Mummendey, A., Klink, A., Mielke, R., Wenzel, M., \& Blanz, M. (1999). Socio-structural characteristics of intergroup relations and identity management strategies: Results from a field study in East Germany. European Journal of Social Psychology, 29(2-3), 259-285.

Mummendey, A., Otten, S., Berger, U., \& Kessler, T. (2000). Positive-negative asymmetry in social discrimination: Valence of evaluation and salience of categorization. Personality and Social Psychology Bulletin, 26(10), 1258-1270.

Niens, N., \& Cairns, E. (2002). Identity Management Strategies in Northern Ireland. The Journal of Social Psychology, 142(3), 371-380. doi:10.1080/00224540209603905

Phinney, J. S. (1990). Ethnic identity in adolescents and adults: Review of research. Psychological Bulletin, 108(3), 499-514.

Sam, D. L., \& Berry, J. W. (2010). Acculturation: When individuals and groups of different cultural backgrounds meet. Perspectives on Psychological Science, 5(4), 472-481. doi:10.1177/ 1745691610373075 
Siverts, H. (2006). Etnicheskaya stabil'nost' i dinamika granits v yuzhnoi Meksike [Ethnic stability and boundary dynamics in Southern Mexico]. In F. Barth (Ed.), Etnicheskie gruppy $i$ sotsial'nye granitsy. Sotsial'naya organizatsiya kul'turnykh razlichii. Sbornik statei [Ethnic groups and social boundaries. The social organization of cultural differences. Collected works] (pp. 122-141). Moscow: Novoe izdatel'stvo. (Transl. of: Siverts, H. (1969). Ethnic stability and boundary dynamics in Southern Mexico. In F. Barth (Ed.), Ethnic groups and boundaries. The social organization of culture difference (pp. 101-116). Boston: Little, Brown and Company).

Sobkin, V. S. (1995). Sravnitel'nyi analiz osobennostei tsennostnykh orientatsii russkikh i evreiskikh podrostkov [A comparative analysis of characteristics of value orientations in Russian and Jewish teenagers]. In Tsennostno-normationye orientatsii starsheklassnika [Value-normative orientations in high school students] (pp. 6-63). Moscow: TsSO RAO.

Stefanenko, T. G. (1999). Sotsial'naya psikhologiya etnicheskoi identichnosti [The social psychology of ethnic identity] (Unpublished doctoral dissertation). Lomonosov Moscow State University, Moscow, Russian Federation.

Stefanenko, T. G. (2014). Etnopsikhologiya: Uchebnik [Ethnopsychology: A textbook] (5th ed.). Moscow: Aspekt Press.

Tajfel, H. (1982). Social psychology of intergroup relations. Annual Review of Psychology, 33, 1-39.

Tajfel, H., Flament, C., Billig, M. G., \& Bundy, R. P. (1971). Social categorization and intergroup behavior. European Journal of Social Psychology, 1, 149-178.

Turner, J. C. (1999). Some current issues in research on social identity and self categorization theories. In N. Ellemers, R. Spears, \& B. Doosje (Eds.), Social identity (pp. 6-34). Oxford: Blackwell Publishing.

Turner, J. C., Brown, R. J., \& Tajfel, H. (1979). Social comparison and group interest in ingroup favoritism. European Journal of Social Psychology, 9, 187-204.

Umaña-Taylor, A. J., Yazedjian, A., \& Bбmaca-Gymez, M. (2004). Developing the Ethnic Identity Scale using Eriksonian and social identity perspectives. Identity: An International Journal of Theory and Research, 4(1), 9-38. doi:10.1207/S1532706XID0401_2

Verkuyten, M., \& Reijerse, A. (2008). Intergroup structure and identity management among ethnic minority and majority groups: The interactive effects of perceived stability, legitimacy, and permeability. European Journal of Social Psychology, 38, 106-127. doi:10.1002/ejsp.395

Verkuyten, M., \& Yildiz, A. A. (2007). National (Dis)identification and ethnic and religious identity: A study among Turkish-Dutch muslims. Personal and Social Psychology Bulletin, 33(10), 1448-1462. doi:10.1177/0146167207304276

Marina V. Kotova - senior lecturer, Faculty of Social Sciences, School of Psychology, Department of Organizational Psychology, National Research University "Higher School of Economics", Ph.D.

Research areas: methodology (research methods, functional approach in psychology), social psychology (social group formation, intergoup perception, attidude structure and functions, social identity, social conflict), ethnic psychology (ethnic prejudice and ethnic identity formation, measurement, and change).

E-mail: mkotova@hse.ru 